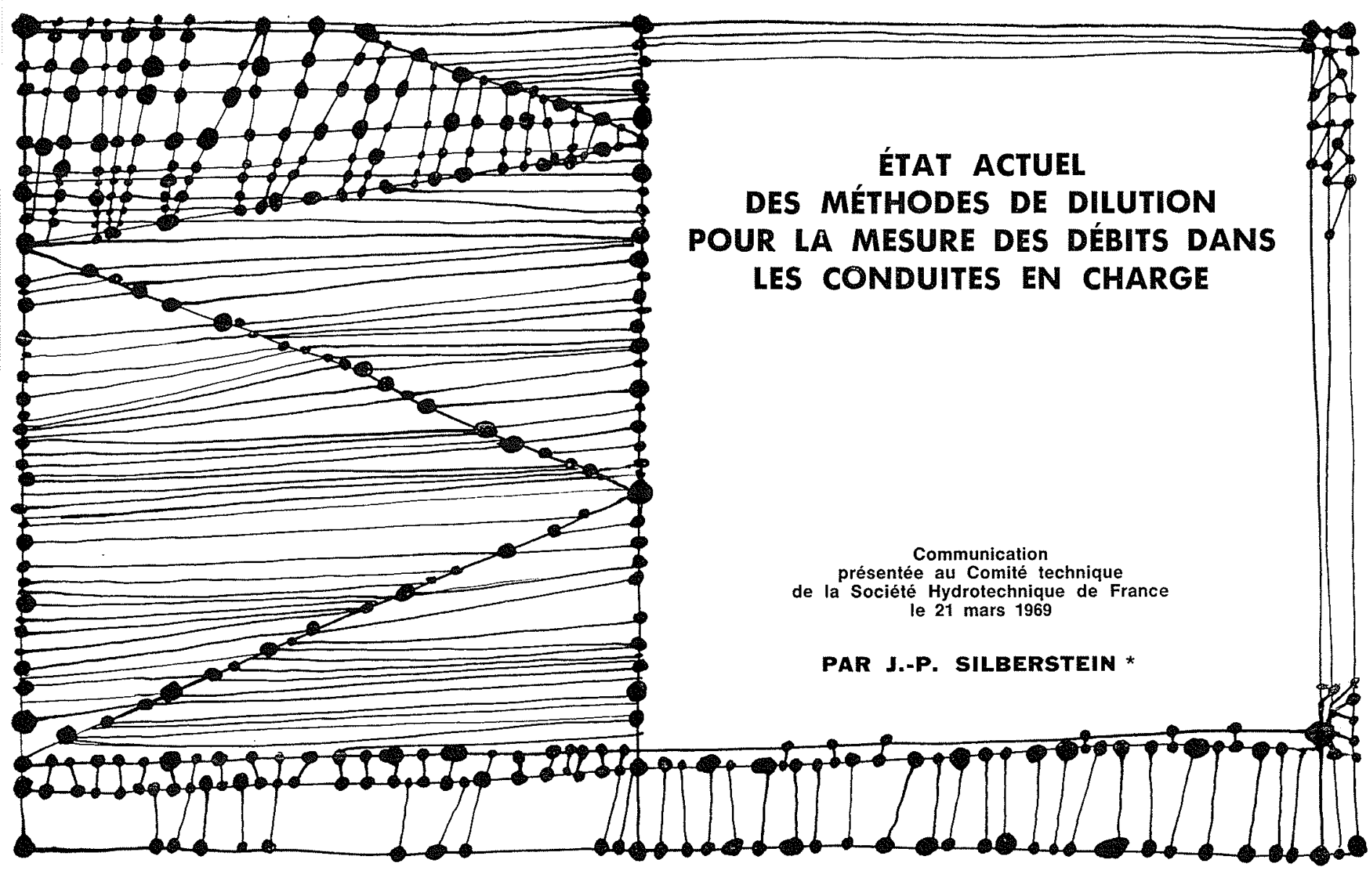

S'il est possible, à l'heure actuelle, de mesurer des grandeurs parfois très difficilement accessibles avec des précisions dépassant souvent l'entendement, il est troublant de constater que la mesure du débit d'un fluide reste toujours une opération difficile.

Obtenir une telle mesure avec une précision relative de 1 \% nécessite dans la plupart des cas une mise en cuvre lourde et coûteuse. Qui plus est, ce matériel, introduit dans la conduite, peut perturber lui-même la grandeur à mesurer.

Seule la méthode résultant de la définition même d'un débit et consistant à mesurer la quantité de fluide écoulé pendant un temps donné, permet d'ac céder à la grandeur débit avec une précision importante. Malheureusement, ce procédé n'est guère utilisable si ce n'est en tant que méthode d'étalonnage en laboraloire.

Dans le cas des conduiles en charge, les méthodes de dilution, encore peu répandues, offrent de nombreux avantages.

Trois méthodes de mesure peuvent ainsi être utilisées. Deux d'entre elles, appelées méthodes d'intégration et méthode d'injection à débit constant, ont ceci en commun qu'elles nécessitent la mesure de la concentration en traceur, en aval de son point d'injection.

Ces deux méthodes utilisées avec des traceurs chimiques ou colorés sont à proscrire dans le cas de mesure en conduite, car elles nécessiteraient de pratiquer des trous dans la conduite aux points de prélèvement. Utilisées avec un traceur radioactif, elles sont là encore difficilement exploitables car, bien que les sondes puissent ètre extérieures à la

* E.D.F., Direction des Etudes et Recherches, Chatou. conduite, la quantité de traceur est difficilement dosable avec précision, et d'autre part, le traceur disparait spontanément dans le temps par décroissance radioactive.

Par contre, une troisieme méthode, celle du nuage radioactif, trouve dans le cas de conduites fermées tout son intérêt. Ici, la quantité de traceur injecté importe peu, pour autant qu'on puisse le détecter dans les sections de mesure.

Préconisée en 1923 par Allen et Taylor, cette méthode a fait, depuis lors, l'objet de nombreuses études tant théoriques qu'expérimentales. Il est d'ailleurs amusant de remarquer que si l'imaginalion des chercheurs s'est révélée fertile en résultats, elle l'a été presque autant sur le plan de la terminologie. Les études faites dans ce domaine portent en effet des titres aussi variés que méthode d'Allen, de l'écran salin, des deux pies, en langue française, de pulse dilution method, peak tuning pour les anglo.saxons.

Nous ajouterons à cette liste déjà longue la méthode du nuage radioactif, car elle nous semble coller mieux à la réalité.

Mais quarante années d'efforts n'ont pas permis aux expérimentateurs el théoriciens de proposer une technique de mesure véritablement opérationnelle. Il manquait un maillon à la chaîne. Ce n'est que tout récemment, avec l'apparition de générateurs de solutions radioactives que la méthode du nuage radioactif a pu révéler ses véritables possibilités. Nous verrons pourquoi lors de la description de ce générateur.

Mais, auparavant, voyons le principe de la méthode.

Supposons qu'il soit possible de déposer dans une section droite d'une conduite une quantité don- 
née de traceur avec une distribution uniforme. Supposons de plus que la vitesse du fluide est constante en tout point de la section et que le traceur ne diffuse pas dans le fluide.

Dès lors, la mesure du temps que met cette tranche de fluide marqué entre deux sections, dites sections de mesure, situées à distance $l$ connue l'une de l'autre, permet de calculer la vitesse du fluide, donc, en supposant connue la section, le débit à mesurer.

Malheureusement, il faut ètre réaliste et tenir compte :

$1^{\circ}$ de la distribution non uniforme de vitesse dans une section;

$2^{\circ}$ de l'impossibilité de marquer une tranche infiniment mince de fluide, avec une répartition homogène de traceur;

$3^{\circ}$ de la diffusion turbulente qui tend à diluer le nuage initial, tant radialement que axialement.

Nous allons montrer néanmoins que si l'on opère la détection du nuage suffisamment loin en aval du point d'injection, d'une part, et si l'on mesure, d'autre part, la vitesse du centre de gravité temporel du nuage, alors, malgré tout, la vitesse ainsi mesurée est bien la vitesse moyenne du fluide, à partir de laquelle on peut donc calculer le débit.

\section{Les équations}

\section{de la diffusion turbulente}

Notations.

$$
\begin{aligned}
& \mathbf{R} \text { rayon de la conduite; } \\
& \text { D diamètre de la conduite; } \\
& r \text { rayon polaire; } \\
& \theta \text { angle polaire; } \\
& x \text { abscisse mesurée le long de la con- } \\
& \text { duite; } \\
& t \text { variable temporelle; } \\
& u \text { vitesse locale; } \\
& \mathrm{U} \text { vitesse moyenne; } \\
& u_{*} \text { vitesse de frottemenl; } \\
& c \text { concentration locale; } \\
& c_{m} \text { concentration moyenne; } \\
& Z=r / \mathrm{R} \text { rayon polaire réduit; } \\
& \xi=x / \mathrm{R} \text { abscisse réduite; } \\
& \xi_{1}=\frac{x-\mathrm{U} t}{\mathrm{R}} \text { abscisse réduite pour un observateur } \\
& \rightarrow \tau=\frac{u_{x} t}{\mathrm{R}} \text { variable temporelle réduite; } \\
& n=\frac{\mathrm{U}}{u l_{*}} \\
& K_{1} \text { coefficient de diffusion radiale; } \\
& \mathrm{K}_{2} \text { coefficient de diffusion tangentielle; } \\
& \mathrm{K}_{3} \text { coefficient de diffusion axiale. }
\end{aligned}
$$

\section{Equation de la diffusion.}

Soit $\vec{A}$ le transfert de matière sortant de la surface entourant un volume élémentaire de concentration $c$, l'équation de continuité s'écrit :

$$
\operatorname{div} \vec{A}+\frac{\partial c}{\partial \tau}=0
$$

$\vec{A}$ résulte d'une part de la convection, d'autre part de la diffusion turbulente.

Les composantes de la convection sont :

- composante radiale : 0 ,

- composante tangentielle : 0 ,

- composante axiale : $\left(u / u_{t}\right) c$.

En fait, on adoptera la valeur moyenne $\left(\mathrm{U} / u_{*}\right) c$.

Les composantes du terme de diffusion turbulente sont proportionnelles au gradient de la concentration :

- composante radiale : $-K_{1}(\partial c / \partial Z)$,

- composante tangentielle : - $\mathbf{K}_{2}(\partial c / Z \partial \theta)$,

- composante axiale : $-\mathbf{K}_{\mathbf{3}}(\partial c / \partial \mathbf{Z})$.

En s'appuyant sur le fait que les systèmes turbulents ont une forte tendance à l'isotropie (Sir G.I. Taylor, "The dispersion of water in turbulent flow through a pipe », «British Hydromechanics Research Association, K. J. Whiteman, mars $1955 \gg$ ), on écrira :

$$
\mathrm{K}_{1}=\mathrm{K}_{2}=\mathrm{K}_{3}=\mathrm{K}(\mathrm{Z})
$$

Si l'on suppose une symétrie axiale $:(\partial c / \partial \theta)=0$ et dès lors :

$$
\operatorname{div} \overrightarrow{\mathrm{A}}=\frac{\partial}{\partial x}\left(\frac{u}{u_{*}} c-K_{1} \frac{\partial c}{\partial x}\right)+\frac{\partial}{\partial Z}\left(-K_{2} \frac{\partial c}{\partial Z}\right)
$$

En négligeant la diffusion axiale devant la convection, l'équation pourra s'écrire :

$1^{\circ}$ dans un système de coordonnées fixes par rapport à la conduite :

$$
\frac{\partial c}{\partial t}+u \frac{\partial c}{\partial x}=\frac{\partial}{\partial Z}\left(\mathbf{K} \frac{\partial c}{\partial Z}\right)
$$

$2^{\circ}$ dans un système de coordonnées se déplaçant a la vitesse moyenne $U$ du fluide dans la conduite :

$$
\frac{\partial c}{\partial t}+\frac{\mathrm{U}}{u_{*}} \frac{\partial c}{\partial \xi}=\frac{1}{\mathrm{Z}} \frac{\partial}{\partial \mathrm{Z}}\left(\mathrm{ZK} \frac{\partial c}{\partial \mathrm{Z}}\right)
$$

\section{Vitesse de déplacement du centre de gravité}

L'expression de l'abscisse temporelle du centre de gravité d'un nuage quelconque, enregistré par une sonde fixée sur la conduite est :

$$
\mathrm{OG}=\frac{\int_{0}^{1} 2 \pi Z \int_{0}^{\infty} c t d t d Z}{\int_{0}^{1} 2 \pi Z \int_{0}^{\infty} c d t d Z}
$$

Supposons, et nous montrerons plus loin que ceci est la réalité, que pour toute valeur de $x$ supérieure à une valeur donnée $\mathrm{L}$, la concentration est uniforme dans une section, soit :

$$
\frac{\partial c}{\partial Z}=0
$$


Appelons $c_{m}$ la valeur de cette concentration moyenne. $c_{m}$ sera donc telle que :

$$
c_{m}=\int_{0}^{1} 2 \mathrm{ZcdZ}
$$

L'équation de la diffusion devient :

$$
\frac{\partial c}{\partial t}+u \frac{\partial c}{\partial x}=0
$$

Multiplions les deux membres par $Z d Z$ et intégrons de 0 à 1 :

$$
\frac{\partial}{\partial t} \int_{0}^{1} Z c d Z+\frac{\partial}{\partial x} c \int_{0}^{1} u Z d Z=0
$$

Soit, en remarquant que :

$$
\mathrm{U}=\int_{0}^{1} 2 \text { u } \mathrm{Z} d \mathrm{Z}
$$

Si U est la vitesse moyenne de l'écoulement :

Posons :

$$
\frac{\partial c_{m}}{\partial t}+\mathrm{U} \frac{\partial c_{m}}{\partial t}=0
$$

$$
f=\int_{0}^{\infty} e^{-\mu t} c_{m} d t
$$

On remarquera que :

$$
\begin{gathered}
f(\mu=0)=\int_{0}^{\infty} \int_{0}^{1} c \mathrm{Z} d \mathrm{Z}=\mathrm{D} \\
\frac{\partial f}{\partial \mu}(\mu=0)=-\int_{0}^{\infty} \int_{0}^{1} \text { ct } \mathrm{Z} d \mathrm{Z}=\mathrm{N}
\end{gathered}
$$

et que dès lors :

$$
\mathrm{OG}=--\frac{\mathrm{N}}{\mathrm{D}}
$$

Cette transformation est celle de Laplace et l'équation (5) devient:

$$
\mu f+\mathrm{U} \frac{\partial f}{\partial x}=0
$$

dont la solution est :

d'où :

$$
f=a e^{\mu\left[\left(x-x_{0}\right) / 0\right]}
$$

avec $x_{0} \geqslant \mathrm{~L}$

$$
\frac{\partial f}{\partial \mu}=-a \frac{x-x_{0}}{-U} e^{\mu\left[\left(x-x_{0}\right) / U\right]}
$$

donc :

$$
\begin{gathered}
f(\mu=0)=a \\
\frac{\partial f}{\partial \mu}(\mu=0)=-a \frac{x-x_{0}}{\mathrm{U}}
\end{gathered}
$$

d'où :

$$
\mathrm{OG}=\frac{x-x_{0}}{\mathrm{U}}
$$

Donc, pour toute valeur de $x>\mathrm{L}$, en aval de laquelle la concentration est homogène dans une section, le centre de gravité temporel du nuage se déplace à la vitesse moyenne U de l'écoulement.
Distance de bon mélange $\mathrm{L}$

Nous avons admis, pour mener à bien le calcul ci-dessus, qu'en aval d'une section située à une distance $L$ du point d'injection, le mélange devenait homogène dans une section. Cette distance, nous l'appellerons distance de bon mélange, et nous allons démontrer qu'elle existe.

Reprenons l'équation de la diffusion, écrite cette fois-ci dans un système d'axes se déplaçant à la vitesse moyenne du fluide dans la conduite:

$$
\frac{1}{Z} \frac{\partial}{\partial Z}\left(Z \mathrm{~K} \frac{\partial c}{\partial Z}\right)=\frac{u}{u_{*}} \frac{\partial c}{\partial \xi}+\frac{\partial c}{\partial \tau}
$$

Supposons que la concentration est la même dans toutes les sections de la conduite $[(\partial c / \partial \xi)=0]$, et que le coefficient de diffusion transversal est parabolique, soit :

$$
\mathrm{K}=k \mathrm{Z}(1-\mathrm{Z})
$$

L'équation (3) devient :

$$
\frac{1}{Z} \frac{\partial}{\partial Z}\left\lceil Z^{2}(1-Z) \frac{\partial c}{\partial Z}\right\rceil=\frac{1}{k} \frac{\partial c}{\partial \tau}
$$

Cherchons une solution de la forme:

$$
c=c_{i n}+e^{-\alpha \tau} \mathbf{F}(Z)
$$

en appelant toujours $c_{m}$ la concentration moyenne dans une section.

L'équation (6) devient (en posant $r=\alpha / k$ ) :

$$
\left(Z-Z^{2}\right) F^{\prime \prime}+(2-3 Z) F^{\prime}+r F=0
$$

Or, on connaît une solution de l'équation différentielle :

$\left(Z-Z^{2}\right) \mathrm{F}^{\prime \prime}+[c-\mathrm{L}(a+b+1) Z] \mathrm{F}^{\prime}-a b \mathrm{~F}=0$

Soit, en prenant $c=2, a+b=2, a b=-r$ :

$$
\begin{aligned}
\mathbf{F}_{n}(Z) & =1-\frac{r}{2} Z-\frac{r(3-r)}{2 \times 3} \frac{Z^{2}}{2 !} \ldots \\
& -\frac{r(3-r)(8-r) \ldots|n(n+2)-r| Z^{n+1}}{(n+2) !}
\end{aligned}
$$

d'où la solution cherchée :

$$
c=c_{m}+\Sigma d_{n} e^{-\alpha_{n} \tau} \mathrm{F}_{n}(\mathrm{Z})
$$

avec $\alpha n=r n / \mathrm{K}$

En exprimant que :

$$
c_{m}=\int_{0}^{1} 2 \pi Z c d Z
$$

on montre que $r$ peut prendre toute valeur de la forme :

$$
r_{n}=n(n+2)
$$

avec $n=1,2,3 \ldots$

Considérons maintenant une distribution initiale de concentration telle que :

$$
\begin{aligned}
& c=\mathrm{A}^{2} \text { pour } 0<Z<1 / \mathrm{A} \\
& c=0 \text { pour } 1 / \mathrm{A}<Z<1
\end{aligned}
$$


On peut aisément établir une condition d'orthogonalité :

$$
\int_{0}^{1} Z \mathrm{~F}_{n} \mathrm{~F}_{p} d Z=0
$$

pour $n \neq p$

qui, avec les conditions initiales choisies, nous permet de calculer :

$$
a_{n}=\frac{A^{2} \int_{n}^{1 / A} Z F_{n}(Z) d Z}{\int_{0}^{1} Z F_{n}^{2} d Z}
$$

Calculons les premiers termes de $c$ et leurs coefficients; et les coefficients des termes d'amortissement :

$$
\begin{aligned}
& \mathrm{F}_{1}=1-\frac{3}{2} Z \\
& \mathrm{~F}_{2}=1-4 Z+\frac{10}{3} Z^{2} \\
& \mathrm{~F}_{3}=1-\frac{15}{2} Z+15 Z^{2}-\frac{10}{3} Z^{3} \\
& a_{1}=8\left(1-\frac{1}{A}\right) \\
& a_{2}=\frac{9}{2}\left(6-\frac{16}{\mathrm{~A}}+\frac{10}{\mathrm{~A}_{2}}\right) \\
& \alpha_{1}=3 \mathrm{~K} \\
& \alpha_{2}=8 \mathrm{~K}
\end{aligned}
$$

Soit, pour différentes valeurs de $A$, les valeurs suivantes :

\begin{tabular}{|c|c|c|}
\hline$A$ & $a_{1}$ & $a_{2}$ \\
\hline 10 & 7,2 & 19,35 \\
100 & 7,92 & 26,275 \\
1000 & 7,992 & 26,928 \\
$\infty$ & 8 & 27 \\
& & \\
\hline$A \rightarrow \infty$ correspond à une injection ponctuelle. \\
\hline
\end{tabular}

En prenant pour $K$ la valeur 0,4 donnée par Taylor, calculons les termes d'amortissement pour différentes valeurs de $\tau$ :

\begin{tabular}{|r|l|l|}
\hline$\tau$ & $e^{-1,2 \tau}$ & $e^{-3,2 \tau}$ \\
\hline 1 & 0,301 & 0,0407 \\
2 & 0,0907 & 0,00166 \\
3 & 0,027 & 0,0000677 \\
10 & 0,00000614 & \\
\hline
\end{tabular}

On constate donc, qu'en pratique, tous les termes sont petits devant le premier et qu'une majorante de son coefficient est 8 .

D'où :

$$
c \simeq c_{m}\left\{1+8[1-(3 / 2) Z] e^{-1,2 \tau}\right\}
$$

Calculons alors l'écart de concentration entre l'axe et la paroi de la conduite:

$$
\Delta c=\frac{c_{0}-c_{1}}{c_{m}}=12 e^{-1,2 \tau}
$$

On constate que cet écart tend exponentiellement vers 0 , ce qui prouve le bien fondé de l'hypothèse de bon mélange.

\section{Expression pratique de $L$}

Ce calcul va nous permettre d'évaluer $L$.

Calculons le temps $\tau$ au bout duguel :

$$
\begin{gathered}
\Delta c=\mathrm{X}^{10^{-2}} \\
12 e^{-1,2 \gamma}=\mathrm{X}^{-10^{-2}}
\end{gathered}
$$

$\mathrm{Or}^{*}:$

$$
\tau=\frac{\mathrm{U} t}{n \mathrm{R}}=\frac{2 \mathrm{~L}}{n \mathrm{D}} \quad \text { si } n=\frac{\mathrm{U}}{u_{*}}
$$

Si L est la distance parcourue par le liquide se déplaçant à la vitesse moyenne $U$ pendant le temps $\tau:$

$$
\begin{aligned}
& 12 e^{-[(1,2 \times 2 \mathrm{~L}) / n \mathrm{D})]}=\mathrm{X} 10-2 \\
& \frac{\mathrm{L}}{\mathrm{D}}=2,9542 n-\frac{n}{2,4} \ln \mathrm{X}
\end{aligned}
$$

Donc, la distance de bon mélange, exprimée en nombre de diamètres est:

$$
\frac{\mathrm{L}}{\mathrm{D}}=2,9542 n-\frac{n}{2,4} \ln \mathrm{X}
$$

soil pour $\mathrm{X}=1$ :

$$
\frac{\mathrm{I}}{\mathrm{D}}=3 n
$$

Il est facile d'établir que si $K_{s}$ est le coefficient de Strickler :

$$
n=0,25 \mathrm{~K}_{s} \mathrm{D}^{1 / 6}
$$

D'autre part, on notera $\lambda$ le coefficient de perte de charge linéaire. Dès lors, la longueur de bon mélange à $1 \%$ près pourra s'écrire :

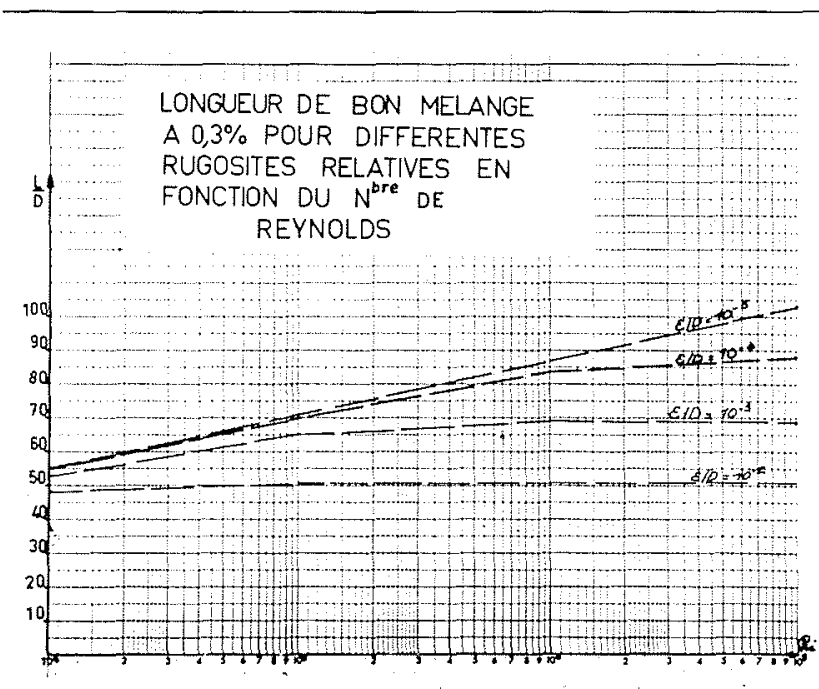

1/ 


$$
\begin{aligned}
& \frac{\mathrm{L}}{\mathrm{D}}=3 n \\
& \frac{\mathrm{L}}{\mathrm{D}}=0,75 \mathrm{~K}_{s} \mathrm{D}^{1 / 6} \\
& \frac{\mathrm{L}}{\mathrm{D}}=3 \sqrt{\frac{8}{\lambda}}
\end{aligned}
$$

Pour des Reynolds variant de $10^{4}$ a $10^{7}$ et pour les rugosités relatives variant de $10^{-2}$ à $10^{-5}$, des courbes permettent de prévoir $L / D$ pour obtenir des mélanges à $0,3,1,5$ \% .

Dans la pratique, les conduites fermées habituel-

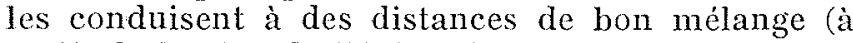
$1 \%$ ) de l'ordre de 75 diametres dans le cas d'une injection centrale.

\section{Erreur systématique de la méthode en fonction de l'homogénéité du mélange}

Considérons que dans la section de mesure choisie, le mélange est réalisé à $\mathrm{X} \%$.

Nous venons de montrer que $c$ peut s'écrire :

et :

$$
c=c_{m}\left[1+8\left(1-\frac{3}{2} Z\right) e^{-1,2 \tau}\right]
$$

$$
\Delta c=12 e^{-1,2 \tau}=\times 10^{-2}
$$

soit :

$$
\begin{aligned}
& c=c_{m}\left[1+8\left(1-\frac{3}{2} Z\right) \frac{\mathrm{X}}{12} 10^{-2}\right] \\
& c=c_{m}\left[1+\frac{2}{3}\left(1-\frac{3}{2} Z\right) \varepsilon\right]
\end{aligned}
$$

en posant $\varepsilon=\mathrm{X}^{10-2}$

La loi de répartilion des vitesses dans la section peut s'écrire d'autre part:

$$
u==\mathrm{U}\left[1+\frac{1}{n k}\left(\frac{3}{2}+\log (1-Z)\right)\right]
$$

Dans ce cas, l'équation différentielle (4) devient:

$$
\begin{array}{r}
\frac{\partial}{\partial t} \int_{0}^{1} Z c d Z+\frac{\partial}{\partial x} \int_{0}^{1} U c_{m} Z\left[1+8\left(1-\frac{3}{2} Z\right) \varepsilon\right] \\
{\left[1+\frac{1}{n k}\left(-\frac{3}{2}+\log (1-2)\right)\right] d Z=0}
\end{array}
$$

Par définition :

$$
c_{m}=\int_{0}^{1} 2 Z c d Z
$$

donc, le $1^{\text {tr }}$ lerme de l'équation est :

$$
\frac{1}{2} \frac{\partial c_{m}}{\partial t}
$$

le 2 terme se calcule aisément et s'écrit :

$$
\frac{1}{2} U \frac{\partial c_{m}}{\partial x}\left(1+\frac{2 \varepsilon}{9 k n}\right)
$$

L'équation (5) s'écrit done:

$$
\frac{\partial c_{m}}{\partial t}+\mathrm{U}\left(1+\frac{2 \varepsilon}{9 k n}\right) \frac{\partial c_{m}}{\partial x}=0
$$

équation identique à (5) qui implique que le centre de gravité du nuage n'a plus la vitesse $U$, mais :

$$
U\left(1+\frac{2 \varepsilon}{9 k n}\right)
$$

Donc, l'erreur de mesure sur U est :

$$
\frac{\Delta U}{U}=\frac{2 \varepsilon}{9 k n}=\frac{2}{9 k n} \times 10^{-2}
$$

Si $n=20$ et $k=0,4$ :

$$
\frac{\Delta \mathrm{U}}{\mathrm{U}} \simeq \frac{1}{36} \times 10^{-2}
$$

Donc, dans une section où l'écart de concentration entre le centre et la paroi est de X\%, l'erreur systémalique sur la mesure de la vitesse moyenne du fluide par détection de la vitesse du centre de gravité temporel d'un nuage marqué est d'environ $\mathrm{X} / 40 \%$ par excès.

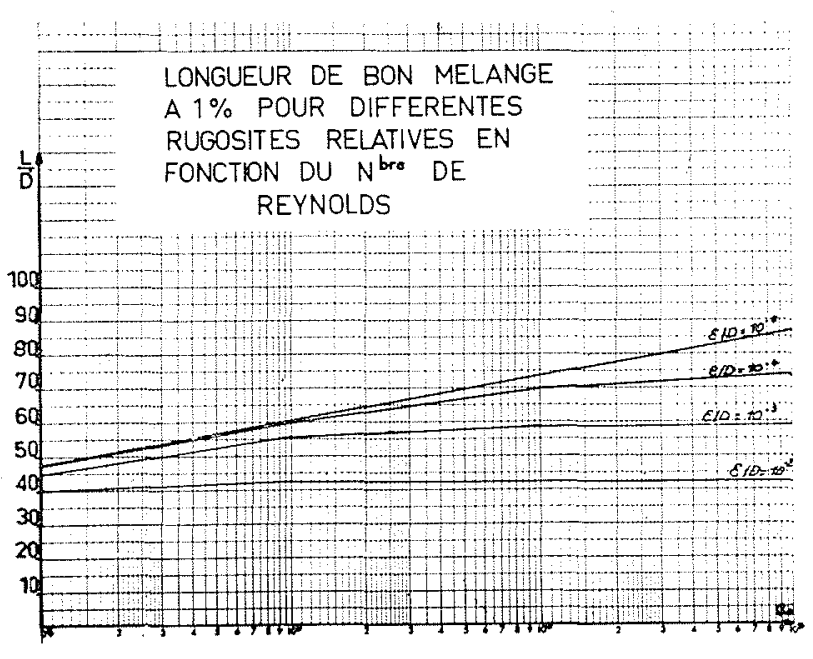

2I

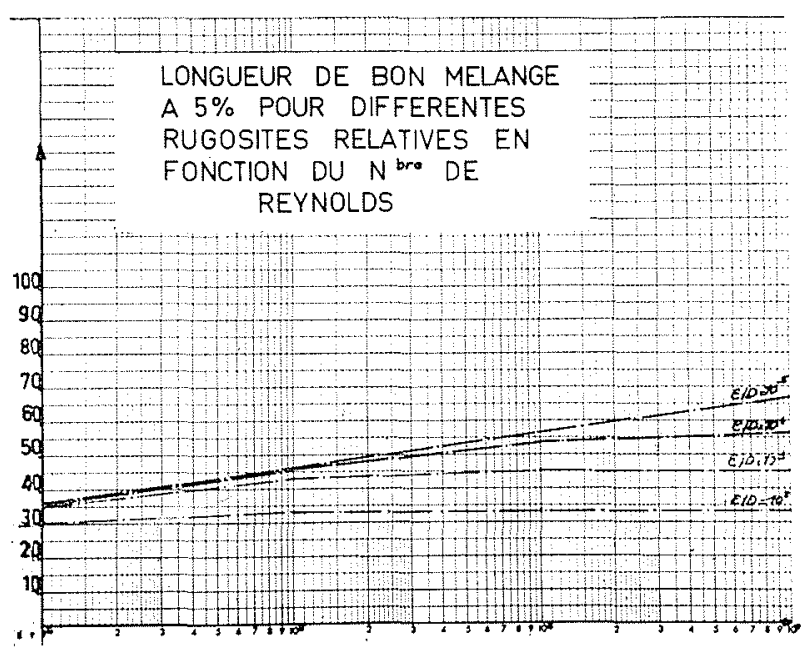

$3 /$ 


\section{Cas de détecteurs extérieurs}

Dans ce cas, il faut considérer l'évolution de la concentration que voit le détecteur, c'est-à-dire faire intervenir un coefficient de pondération $p(x, z, \theta)$ fonction de l'angle solide qui intéresse le détecteur et de l'auto-absorption du rayonnement émis par le traceur, par le fluide lui-mème.

Dès lors, l'abscisse temporelle du centre de gravité d'un nuage sera :

$$
O\left(x^{\prime}=\frac{\int_{\mathrm{V}} \int_{0}^{\infty} p c t d t d v}{\int_{\mathrm{V}} \int_{0}^{\infty} p c d t d v}\right.
$$

Soit :

$$
O \mathrm{G}^{\prime}=\frac{\int_{\mathrm{v}} p\left[\left(x-x_{0}\right) / u\right] d \nu}{\int_{\mathrm{v}} p d v}
$$

D'après les calculs précédents:

$$
\begin{aligned}
& \int_{0}^{\infty} c d t=a \\
& \int_{0}^{\infty} c t d t=a \frac{x-x_{0}}{u}
\end{aligned}
$$

Considérons deux sondes situées à distance $l$ l'une de l'autre, positionnées aux points d'abscisse $\xi_{1}$ et $\xi_{2}$, et géométriquement semblables vis-à-vis de l'écoulement.

On posera : $x=\xi+\alpha$ :

$$
\begin{aligned}
& \mathrm{OG}_{2}-\mathrm{OG}_{1}= \\
& \frac{\int_{V} a p \frac{\xi_{2}+\alpha-x_{0}}{u} d v-\int_{V} a p \frac{\xi_{1}+\alpha-x_{0}}{u} d v}{\int_{V} a p d v}
\end{aligned}
$$

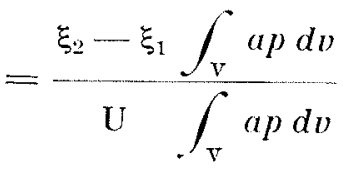

$$
\begin{aligned}
& \frac{\int_{V} a p \alpha d v-\int_{V} a p \alpha d v}{\int_{V} a p d v} \\
& =\frac{\xi_{2}-\xi_{1}}{u}=\frac{l}{\mathrm{U}}
\end{aligned}
$$

Donc, le résultat est conservé : la vitesse de déplacement du centre de gravité mesurée entre les deux sondes, supposées semblables, est la vitesse moyenne du fluide dans la conduite.

\section{Pratique de la méthode}

Dans les développements précédents, il n'a pas été fait état de la qualité du traceur, mais il a été implicitement admis que l'on devait mesurer l'évolution dans le temps de la concentration moyenne dans une section de la conduite.
L'utilisation d'un produit chimique comme traceur, ou d'un colorant nécessiterait de percer la conduite pour pouvoir mesurer les concentrations. Bien au contraire, si le traceur est un radioélément, il est possible, si celui-ci est bien choisi, d'en détecter la concentration à travers la conduite.

Le choix de ce radiójément doit être fait en fonclion des impératifs suivants :

- énergie suffisante pour pouvoir être détecté à travers la conduite;

période suffisamment longue pour avoir le temps de faire la mesure, suffisamment courte pour que l'activité totale injectée soit ramenée à une valeur inférieure à la C.M.A. légale avant rejet. Jusqu'à ces dernières années, le problème était difficile à résoudre. Les radioéléments utilisés devaient être transportés de leur lieu de fabrication au lieu d'utilisation et cela nécessitait de les choisir avec des périodes relativement longues, de prévoir de plus, les dates des essais en fonction des dates de fabrication, d'utiliser des quantités faibles puisque, entre l'instant d'utilisation et le moment du rejet du fluide à mesurer, la quantité injectée n'avait, vu la période radioactive assez grande, pas le temps de décroître de façon notable.

Malgré ces difficultés, de nombreux essais ont été faits, mettant en jeu des radioéléments tels que l'I $\mathrm{I}_{131}$, le $\mathrm{Br}_{82 .}$, le $\mathrm{Na}_{24}$. Mais les difficultés opératoires restaient grandes et l'intérêt de la méthode, limité.

L'apparition, il y a quelques années, de généraleurs de radioéléments à période courte a permis de redonner un essor à cette méthode.

Le principe de ces générateurs (parfois appelés «vache ») est très simple. Sur une résine échangeuse d'ions est déposé un radioélément «père »à période radioactive grande. Ce « père »donne par filiation un radioélément de période courte. Le résine est choisie de telle sorte qu'un éluant qui la traverse entraîne le seul élément « fils ».

Plusieurs couples Père-Fils sont envisageables et parmi ceux-ci, nous avons utilisé essentiellement le couple Cs - Ba. Dans celui-ci, la période du Père $\left(\mathrm{Cs}_{137}\right)$ est d'environ trente ans, celle du Fils $\left(\mathrm{Ba}_{137}\right)$ de deux minutes et demie.

Ces chiffres sont très intéressants car la longévité de la cartouche est fonction de la période de l'élément Père (donc nous disposons d'un appareil pouvant fonctionner longtemps sans recharge), et la période de l'élément Fils est pratiquement l'idéal pour les mesures à effectuer. En effet, le temps total d'une mesure entre l'instant de l'injection, et la détection du nuage dans la deuxième section de mesure est dans la pratique comprise entre quelques dizaines de secondes et quelques minutes.

De plus, on calcule qu'un quart d'heure après l'essai, l'activité restante est ramenće à $1 / 1000$ de l'activité injectée. Dans le cas cité du couple Cs - Ba, l'éluant peut être de l'eau ou, pour des problèmes d'encrassement de filtres, du $\mathrm{CO}_{3} \mathrm{Ca}$, produit à très bon marché et sans aucun danger.

Un autre couple est disponible si les temps de mesures doivent être beaucoup plus grands : le couple $S_{n}$ - In. La période de l'élément Fils est de l'ordre de une heure et demie. Mais, dans ce cas, le Père a une période de quatre mois, ce qui nécessite une recharge fréquente de la cartouche et, de plus, l'éluant doit être une solution décinormale d'acide 


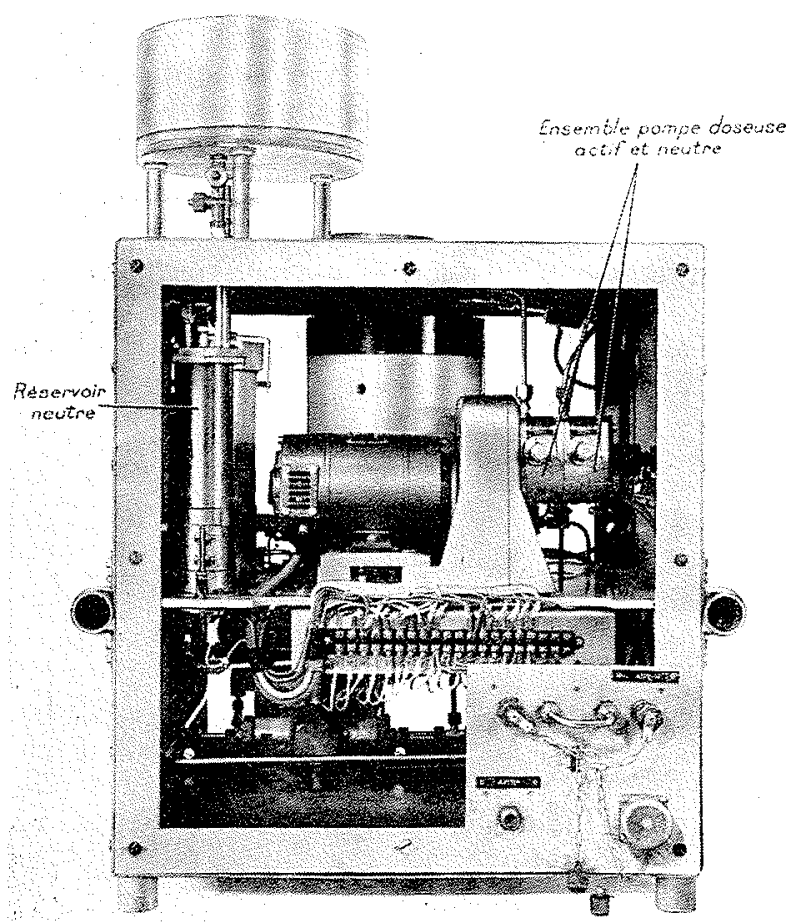

$4 /$
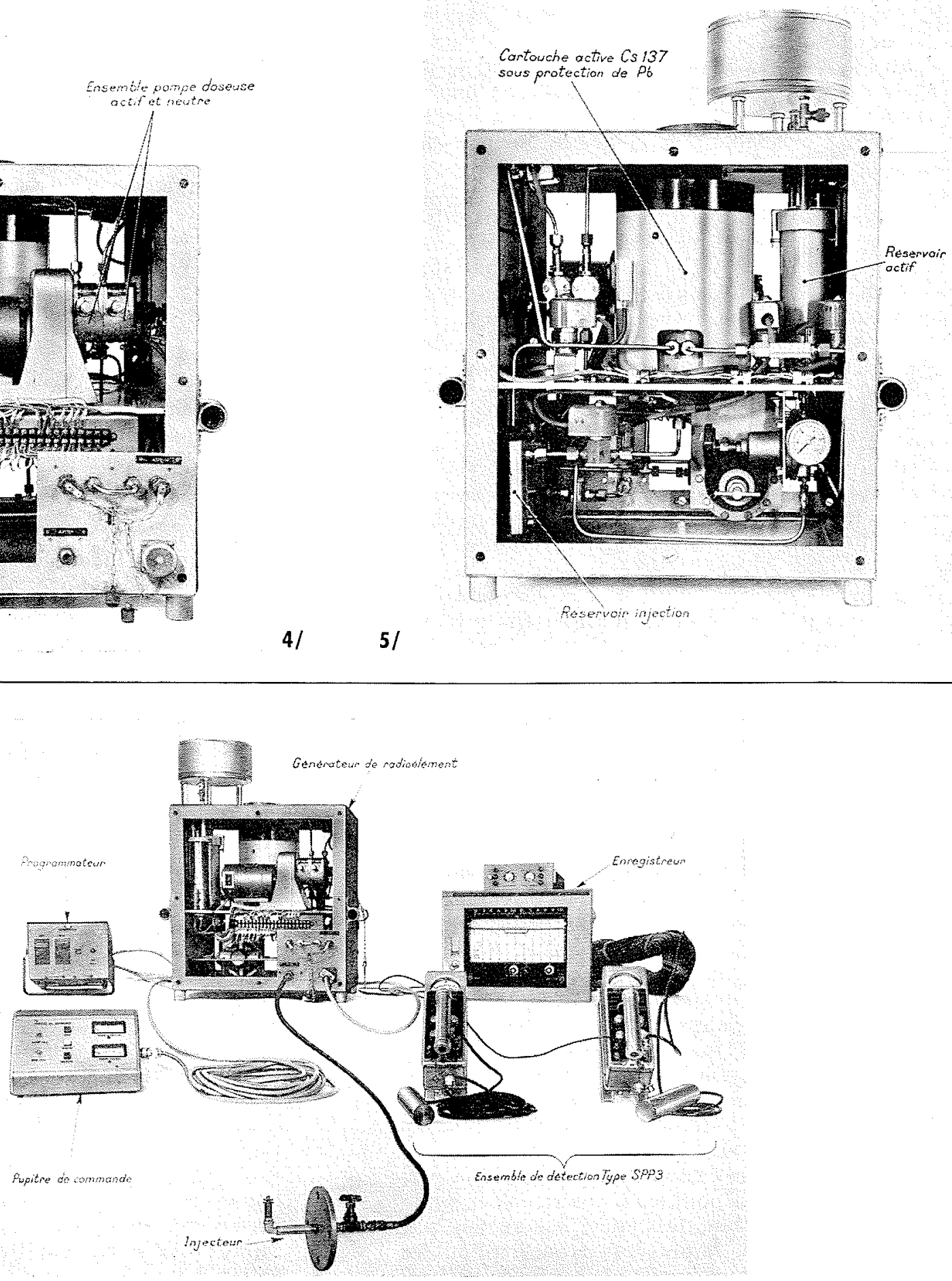

chlorhydrique, ce qui présente des inconvénients. Quoi qu'il en soit, voyons le schéma de fonctionnement d'un tel générateur.

Une pompe doseuse fait circuler, en permanence, le liquide éluant à travers la résine chargée en élément'Père. Lorsqu'une "traite » est désirée, ce liquide, chargé d'éléments Fils est dirigé par une électrovanne dans un réservoir auxiliaire.

Le nombre de coups de pompe, que l'on peut prédéterminer, définit le volume qui sera donc stocké. Quand ce nombre est atteint, une autre électrovanne permet d'introduire une quantité de liquide neutre dans le réservoir auxiliaire pour augmenter le vo- lume de la solution active disponible. Dès lors, se trouve prêt à être utilisé un volume fixé de solution, d'activité globale prévue.

Le réservoir auxiliaire débouche à travers une électrovanne dans un tube flexible relié à l'injecteur. Celui-ci est constitué par une soupape tarée à une faible pression.

La mise à l'air libre du réservoir auxiliaire en même temps que l'ouverture de la vanne située audessous de lui permet à la solution de descendre par gravité jusqu'à la soupape. Une pression d'azote introduite dans le réservoir pousse alors la solution dans la conduite. Cette injection est donc bru- 
tale, sans être cependant infiniment brève, mais on doit remarquer que la détection du nuage sera d'autant plus aisée que le nuage est bref pour une activité donnée.

Toutes ces opérations sont automatiques; le pupitre de commande comportant la mise en marche du générateur, le départ du cycle d'injection, et quelques voyants permettant de suivre le bon déroulement des opérations, se trouve à une dizaine de mètres du générateur, mettant l'opérateur à l'abri de l'inévitable mais faible dose du rayonnement issu du générateur pendant son fonctionnement.

Il faut noter en effet que les pompes étant stoppées, cette dose est inférieure au bruit de fond généralement observé dans nos régions.

Une précaution supplémentaire est de s'assurer qu'aucune quantité d'élément Père ne s'échappe du générateur. Celui-ci, vu sa période importante, risquant de contaminer d'une manière non négligeable et surtout durable, le fluide se trouvant dans la conduite.

Des dosages ont été faits montrant qu'après une dizaine d'injections, l'activité dans l'eau circulant en circuit fermé était 1000 fois inférieure à la C.M.A.

Cet appareil présente donc toutes les garanties quant à un risque éventuel de contamination radioactive on toxique.

Examinons maintenant les appareils de détection. Ceux-ci sont constitués de détecteurs à scintillations. En effet, ce type est le plus adapté, permettant, outre un bon rendement, de ne pas être influencé par les inévitables rayonnements parasites, en éliminant tout complage de particules d'énergie inférieure à celle du traceur utilisé.

Ces sondes sont reliées à un boîtier autonome fonctionnant sur piles et capable d'une part de fournir l'alimentation de la sonde, d'autre part, d'intégrer les impulsions recues dans un ictomètre linéaire à plusieurs gammes.

Les sorties des ictomètres, branchées sur un enregistreur potentiométrique à denx pistes, permettent l'enregistrement des deux nuages. Une base de temps s'inscrit en même temps sur cet enregistrement et permet la mesure du temps écoulé entre le passage des centres de gravité des deux nuages.

Un enregistrement simultané des impulsions délivrées par les détecteurs sur bande magnétique permet d'autre part, de dépouiller a posteriori, et d'une facon plus fine, les résultats. Ceci est réalisé par échantillonnage de ces impulsions, transcription des valeurs des échantillons sur bande perforée, et traitement par calculateur.

Un ensemble de dépouillement automatique est en cours de réalisation qui permettra de délivrer la valeur du temps de propagation du centre de gravité, sur les lieux mêmes de la mesure et dans un temps égal une fois et demie le temps mis pour faire la mesure elle-même.

De nombreuses mesures de débits ont été réalisées à ce jour, dans des conditions plus ou moins favo- rables, permettant de confirmer les résultats théoriques exposés.

Tout d'abord, des mesures ont été faites sur un circuit expérimental de diamètre $350 \mathrm{~mm}$ muni d'un dispositif de mesure de débit de précision 1/1000. Sur ce circuit, nous avons pu vérifier :

$1^{\prime}$ la validité de l'hypothèse sur la distance de bon mélange;

2" l'erreur commise sur la mesure de débit. Cette erreur totale, dans de bonnes conditions de mesure, c'est-à-dire au-delà de la distance de bon mélange était toujours inférieure ou égale à $1 \%$.

Des essais ont été faits de plus :

- sur la conduite forcée de Sassenage $(\varnothing=900)$ et ont conduit à des résultats entachés d'une erreur de $1 \%$;

- sur la conduite rectangulaire de la turbine pompe alimentaire de la centrale de Porcheville $B$, conduite ayant une section d'environ $10 \mathrm{~m}^{2}$, débitant à pleine charge $22 \mathrm{~m}^{3} / \mathrm{s}$ environ.

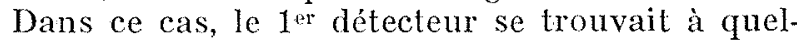
ques diamètres du point d'injection et les résultats ont été entachés d'une erreur de l'ordre de $6 \%$ par rapport à des mesures faites à l'aide d'une batterie de moulinets.

Nous avons été amenés, pour des cas pratiques à vérifier l'influence de coudes pouvant se trouver, soit entre le point d'injection et la $1^{\text {re }}$ section de mesure, soit entre les deux sections de mesure. Le $1^{\text {er }}$ cas offre un avantage, c'est de diminuer la distance de bon mélange d'environ 20 diamètres. Quant au deuxième cas, coude entre les deux sections de mesure, aucune influence sur la précision de la méthode n'a pu être décelée.

Un cas pratique de mesure de débits dans des tubes de faible diamètre (1 pouce), alimentés à partir d'un collecteur de diamètre 200 ou $300 \mathrm{~mm}$, nous a conduit à injecter le traceur dans le collecteur, et à placer les sondes de mesures sur le petit tube. La mesure est alors très facile à réaliser, sans introduction de perte de charge dans le tube, et la précision obtenue de l'ordre de 1 ou $2 \%$.

Ainsi, cette méthode de mesure restée longtemps inexploitée par manque de commodité, est redevenue, avec le générateur de radioéléments, une méthode de mise en ouvre facile, donnant des débits avec une précision semblable à celle donnée par les appareils classiques. Elle offre, sur ces appareils classiques, de nombreux avantages dont les plus importants sont : facilité de mise en œuvre, application à toutes sortes de liquides, possibilité de mesurer des débits dans les nombreux petits tuyaux des installations industrielles modernes, tels les tubes de réfrigération de caissons des réacteurs nucléaires dont on ne savait jusqu'alors équilibrer les débits, échangeurs, tubes écrans des générateurs de vapeur. Un projet de recommandation de l'ISO est d'ailleurs en cours de rédaction, qui précisera les règles correspondant aux meilleures conditions d'emploi. a-

la

is. it, an c-

$\grave{a}$ ps$$
\text { e }
$$$$
\text { !- }
$$

e

$$
\mathrm{t}
$$


Discussion

M. le président ouvre la séance, dont il rappelle lordre du jour, et donne la parole à M. Srbmensteis.

M. le Président remercie M. Sirbersters de son intéressant exposé qui ouve des horizons nouveaux dans le domaine des mesures de dibits dans les conduites.

M. Dollfus demande si l'on enregistre sur la bande du magnétophone un ensemble de sigaux donnant une « base de temps $\gg$.

M. Sumensteix répond que l'on a utilisé un magnetophone dont la vitesse était bien constante. Il signale que, dans un autre cas, pour un enregistrement analogique, on a ajoule au dispositif decrit un système inserivant sur la bande une «base de temps » afin de pallier toute irrégularité éventuelle du déroulement de celle-ci.

M. LIÈvire voudrait savoir si la position du point d'injection dans la conduite a une influence sur la précision de la mesure.

Le point d'injection peut être choisi n'importe ò, dit M. Simberstein, par exemple à l'emplacement d'un trou d'homme de la conduite.

M. Bracher pose les deux questions sujvantes:

1. Quel est le coût d'une opération de mesures, exécutéc par la méthode décrite?

2. A-t-on comparé cette méthode à celle qui consisterait à utiliser des ultra-sons (si la précision recherchée n'est pas aussi grande que celle obtenue par la méthode de dilution)?

M. Silberstein répond :

1. Le coût de l'opération n'a pas été estimé; on sait seulement que le générateur à radjo-éléments revient actuellement a $20000 \mathrm{~F}$ environ et qu'il peut fonctionner trente ans sans recharge (la charge ne coûte que 1000 à $2000 \mathrm{~F}$ ).

2. Aucune comparaison n'a été faite avec les méthodes de mesures par ultra-sons; ces dernières ont l'inconvénient de necessiter l'implantation dans la conduite de deux on quatre générateurs ot capteurs d'ultri-sons (selon les methodes).
M Parsy pose a $M$. Siluersters le problème suivant :

«Il s'agit d'une double conduite de 700 de diamètre, d'environ $17 \mathrm{~km}$ de longueur et de 80 ans d'âge (done vaisemblablement avec fuites), dont on connait seulement les deux extrémités; les pompes sont anciennes et les rendements ne sont pas ceux d'origine. Comment peut-on connaitre les dábits actuels et le débit consécutif à l'installation d'une nouvelle pompe?

M. Silizerstern pense que si l'on peut opérer sur une longueur de conduite d'une centaine de mètres, située vers l'aval, la mesure est possible, entre deux points donnés, le point d'injection étant disposé en tête; les nuages seront extrêmement dilues mais la méthode reste encore valable. La question pourra sans doute etre reprise au cours de la discussion de la communication de M. Axbré.

Pour remédier dans une certaine mesure a l'inconvénient signalé par M. Shlbersters résultant du prix élevé de la méthode d'Allen ave l'utilisation de traceurs radioactifs, M. CARlien rappelle qu'à l'ovigine, la méthode de l'écran salin etait utlisée avec des solutions salines, le passage du nuage étant repéré par la mesure de la conductivité dans les sections limitant le troncon etudié. L'utilisation de cette technique nécessite évidemment d'introduire des électrodes de mesure à lintérieur de la canalisation, mais ceei est souvent facilité par l'existence de tampons de visite ou d'orifices de purge ou de vidange. On evite alors l'utilisation du génerateur de radio-éléments et la méthode devient très économique sans perdre ses avantages de rapidité et de précision.

M. Sinerstre confirme que la méthode classique, encore utilisée, conduit à des résultats du mème ordre que ceux obtenus par la méthode radio-active. L'avantage de cette dernière est de ne nécessiter aucune ouverture dans la conduite en essai.

M. Domergye estime que la mesure des débits par nuages radioactifs présente, par rapport à la mesure chimique. l'avantage de ne pas etre sensible à la turbidité des eaux; ce qui est confirmé par M. Sirbiersteix.

\section{Abstract \\ Instantaneous full-pipe discharge measurement by dilution methods by J.P. Silberstein *}

The radio-active tracer method (one of the three in general used today) has become increasingly popular since the advent of radio-active solution generators. Research in this field of recent years has produced a lot of data which it has become urgent to synthesize. Mathematical proofs are supplied for the following:

(i) Movement of the radio-active "cloud's" centre of gravity at the average flow velocity.

(ii) The existence of a systematic measurement error depending on homogeneity of the mixture.

(iii) The definition of mixing distance beyond which the mixture in a cross-section is homogeneous.

(iv) Methods of estimating the mixing distance.

This method needs special equipment for its application, the main components of which are the radio-element generator, the injection and detection systems and recording and data-abstracting arrangements. The method is briefly described, with results obtained in various-sized pipes. 


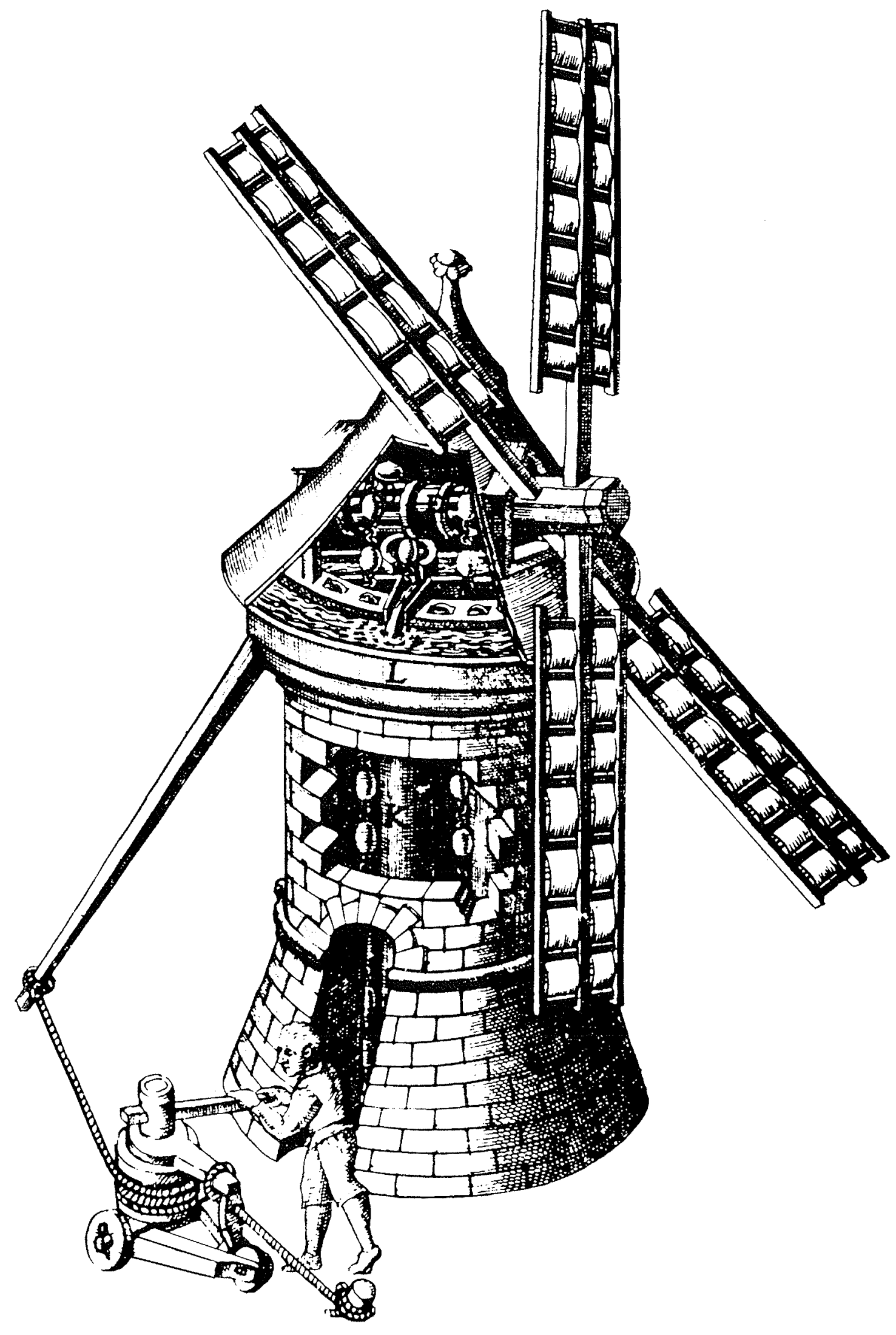

